Ann. Biol. anim. Bioch. Biophys., I969, 9 (I), 9I-99.

\title{
LES GLYCEROPHOSPHATIDES \\ DE LOCUSTA MIGRATORIA CINERASCENS (FABR.) \\ ET DE SON MUTANT ALBINOS (ORTHOPTËRES, ACRIDIDAE), COMPARÉS A CEUX DES AUTRES INSECTES (1)
}

\author{
A. BOUTHIER et R. DOUCE \\ Laboratoire de Zoologie, \\ École normale supérieure, 24 Rue Lhomond, Paris $5^{\mathrm{e}}$ \\ Laboratoire de Biologie vigétale 4 , \\ Faculté des Sciences, 12 Rue Cuvier, Paris 5e
}

\section{SOMMAIRE}

L'étude des phospholipides du tégument abdominal et de l'œil de Locusta migratoria cinerascens FABR. a été réalisée après marquage au ${ }^{32} \mathrm{P}$-orthophosphate de $\mathrm{Na}$ pour comparaison entre une souche normale et une souche mutante albinos. Il n'a pas été trouvé de différence notable entre les deux souches. Les pourcentages des différents constituants de la fraction phospholipidique ont été déterminés par mesure de leur radioactivité après chromatographie sur papier. La composition en phospholipides se rapproche de celle de la majorité des insecte; étudiés jusqu'ici (phosphatidylcholine prédominante sur la phosphatidyléthanolamine), différant donc en cela des Diptères et des Homoptères. Le phosphatidylinositol est présent en quantité d'une importance inédite chez les insectes.

\section{INTRODUCTION}

Les phospholipides des Insectes sont relativement mal connus, car leurs techniques d'étude ne sont parfaitement au point que depuis peu d'années. Les premiers auteurs se sont bornés à préciser le pourcentage des phospholipides par rapport aux lipides totaux (voir la mise au point de FAST, I964). Des travaux plus récents (BIEBER et al., I96I ; ChOJNACKI et KORzYBSKI, I962; CRONE et BRIDGES, I962 ; HAS-

(1) Ce travail a fait l'objet d'une communication à la Euchem Conference on Insect Chemistry, Varenna (Italie), I 3- 77 septembre 1966. 


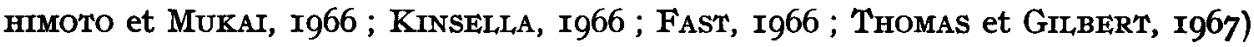
ont montré que les Insectes possèdent dans des proportions variées les différents glycérophospholipides classiques. Tous ces travaux sont relatifs aux Insectes Holométaboles.

L,es phospholipides des Insectes Hétérométaboles ont été beaucoup moins étudiés. Parmi les rares travaux qui leur sont consacrés, citons en particulier ALBREcht (I96I) et Osman et Schmid (I96I) qui ont établi respectivement sur Schistocerca gregaria et sur Locusta migratoria (Orthoptères) le pourcentage des phospholipides dans les lipides totaux; AlIArs et al. (I964) et KINSELLA (I966) qui se sont attachés à l'étude de la cinétique des phospholipides au cours du développement embryonnaire respectivement chez Locusta migratoria et Periplaneta americana. En dehors des travaux de FAST (I 966 et I967), on trouve quelques indications fragmentaires sur deux espèces de Dictyoptères obtenues par CRONE et BRIDGES (I962) en utilisant le marquage au ${ }^{32} \mathrm{P}$, seule méthode permettant de chiffrer avec suffisamment d'exactitude les pourcentages des différents phosphatides.

Outre l'intérêt que présente la connaissance de la composition de la fraction phospholipidique des tissus d'un insecte hétérométabole, l'étude de Locusta migratoria cinerascens à ce point de vue nous a semblé souhaitable du fait de l'apparition récente d'une mutation albinos (VERDIER, I965). Cette mutation pourrait avoir un retentissement corrélatif à l'échelle cellulaire sur les proportions respectives des différents phospholipides. On sait, en effet, que ces constituants tiennent une place primordiale dans 1'architecture lamellaire des diverses membranes cellulaires (DALINER, SIEKEvirz et PALADE, I966). La localisation préférentielle des pigments dans des structures membranaires est établie (FUGE, I966; SHOUP, I966). Ce fait inclinerait à penser que les modifications dans les teneurs en ommochromes et en ptérines (BouTHIER, I966) vont de pair avec des modifications des structures membranaires phospholipoprotéiques des granules qui les contiennent. Ces modifications pourraient être analogues à celles décrites par SHour (I966) chez certains mutants de Drosophila melanogaster. Aussi avons-nous été conduits, dans le cadre des recherches que nous poursuivons sur le déterminisme de l'albinisme chez Locusta, à étudier la composition en phospholipides du tégument abdominal, niveau présentant les différences pigmentaires les plus marquées entre les souches normale et mutante (BouthiER, r966), et de l'œil où les différences sont moins accentuées (id.).

\section{MATÉRIEL ET MÉTHODES}

Trente Locusta migratoria cinerascens ( 5 appartenant à la souche normale, élevée au laboratoire à partir de géniteurs provenant de Castel Sardo (Sardaigne), et 15 à la souche mutante, à raison de 8 femelles et 7 mâles par lot) ont été nourris durant 8 jours avec des feuilles de Phragmites communis $\mathrm{L}$., sur lesquelles était pulvérisée une solution aqueuse d'orthophosphate ${ }^{22} \mathrm{P}$ de $\mathrm{Na}$ correspondant au total à une radioactivité de $2,5 \mathrm{mCi}$. On a ainsi réalisé un marquage uniforme des animaux.

La méthode d'étude des phospholipides est celle mise au point par l'un de nous (DouCE, 1964) sur les tissus végétaux en culture. Les poids frais de tissus utilisés pour l'extraction étaient respectivement de $3 \mathrm{~g}$ pour le tégument abdominal de la souche normale, $2,7 \mathrm{~g}$ pour celui de la souche albinos, et $0,3 \mathrm{~g}$. pour les yeux, les échantillons étant prélevés suivant la technique décrite dans une précédente note (BouTHIER, I966). Après broyage à froid dans un mortier avec du sable en présence 
de méthanol, on porte à ébullition pendant un temps très court, puis on extrait les phosphatides à ${ }^{2} 0^{\circ} \mathrm{C}$ et sous azote par le mélange chloroforme/méthanol $(2: \mathrm{I})$. On élimine ensuite les éléments radioactifs non lipidiques par la méthode de FolCH (1957). L'extrait est alors évaporé à sec sous azote, puis repris dans du chloroforme.

Des chromatogrammes sont réalisés selon la méthode de MaRiNeTti (1957) sur papier. Whatman $n^{0} 1$ imprégné de gel de silice, en phase solvante ascendante dans le mélange di-iso-butyl cétone/acide acétique/eau $(8: 5: \mathrm{I})$. La révélation des phospholipides est effectuée par autoradiochromatographie puis par coloration à la rhodamine $6 \mathrm{G}$ et examen en lumière ultraviolette, ou à l'acide osmique ou encore avec un réactif du phosphore (solution de molybdate d'ammonium dans l'acide perchlorique en milieu acétonique).

Pour plus de sûreté, des désacylations ménagées, selon la méthode de BENSON (I958) modifiée, sont pratiquées pour obtenir les esters phosphorés qui sont alors identifiés en fonction de leur Rf après chromatographie bidimensionnelle en phase solvante ascendante : phénol/eau (100:38) puis méthanol/acide formique/eau $\left(80: x_{3}: 7\right)$.

Pour évaluer les fractions de la quantité totale de phospholipides rapportables respectivement à chaque phosphatide, des mesures de radioactivité ont été faites sur chacune des taches séparées selon la méthode de Marinetri. Pour contrôle, on a effectué des dosages chimiques du phosphore après élution des différents composés présents sur le chromatogramme. Les résultats concordent parfaitement avec les mesures de radioactivité. Il n'a été tenu aucun compte séparé des plasmalogènes correspondant aux différents phospholipides azotés.

Les chromatographies ont été développées conjointement avec des phospholipides témoins qui avaient été antérieurement extraits respectivement du cœur de bœuf pour le cardiolipide (diphosphatidylglycérol), du jaune d'œuf pour la phosphatidylcholine (lécithine), la phosphatidyléthanolamine et la phosphatidylsérine (céphalines), du germe de blé pour le phosphatidylinositol et enfin de la feuille d'épinard pour le phosphatidylglycérol.

\section{RÉSULTATS}

Les résultats présentés par les figures I et 2 sont réunis dans les tableaux I et 2 . On peut tirer certaines conclusions de l'examen de ces tableaux. Le tégument de Locusta est riche en phosphatidylcholine (près de la moitié de la fraction phospholipidique) et en phosphatidyléthanolamine (près du quart de la fraction). Les autres phospholipides (phosphatidylinositol, phosphatidylsérine, cardiolipide et phosphatidylglycérol) existent en plus faible quantité. La présence de lysophosphatidylcholine, bien qu'en faible proportion, a pu être décelée et est très certainement imputable à une phospholipase A qui attaquerait la phosphatidylcholine ou les autres phospholipides azotés pendant le processus d'extraction.

Au niveau oculaire, on constate une exagération des disproportions entre les phosphatides : phosphatidylcholine (plus de $50 \mathrm{p}$. IOo) et phosphatidyléthanolamine (plus de $30 \mathrm{p}$. I00) forment à elles seules près de $85 \mathrm{p}$. Ioo de la fraction phospholipidique, phosphatidylinositol et phosphatidylsérine sont en quantité moindre que dans le tégument; le phosphatidylglycérol paraît absent, alors qu'il existait à l'état de traces dans le tégument, tandis que le cardiolipide montre peu de variation.

D'une manière générale, les teneurs respectives du tégument abdominal et de l'œil en les différents phosphatides sont très voisines les unes des autres dans les deux souches (normale et mutante). Les légères différences que l'on peut relever entre elles sur chacun des tableaux sont peu significatives. On peut facilement imaginer qu'un 


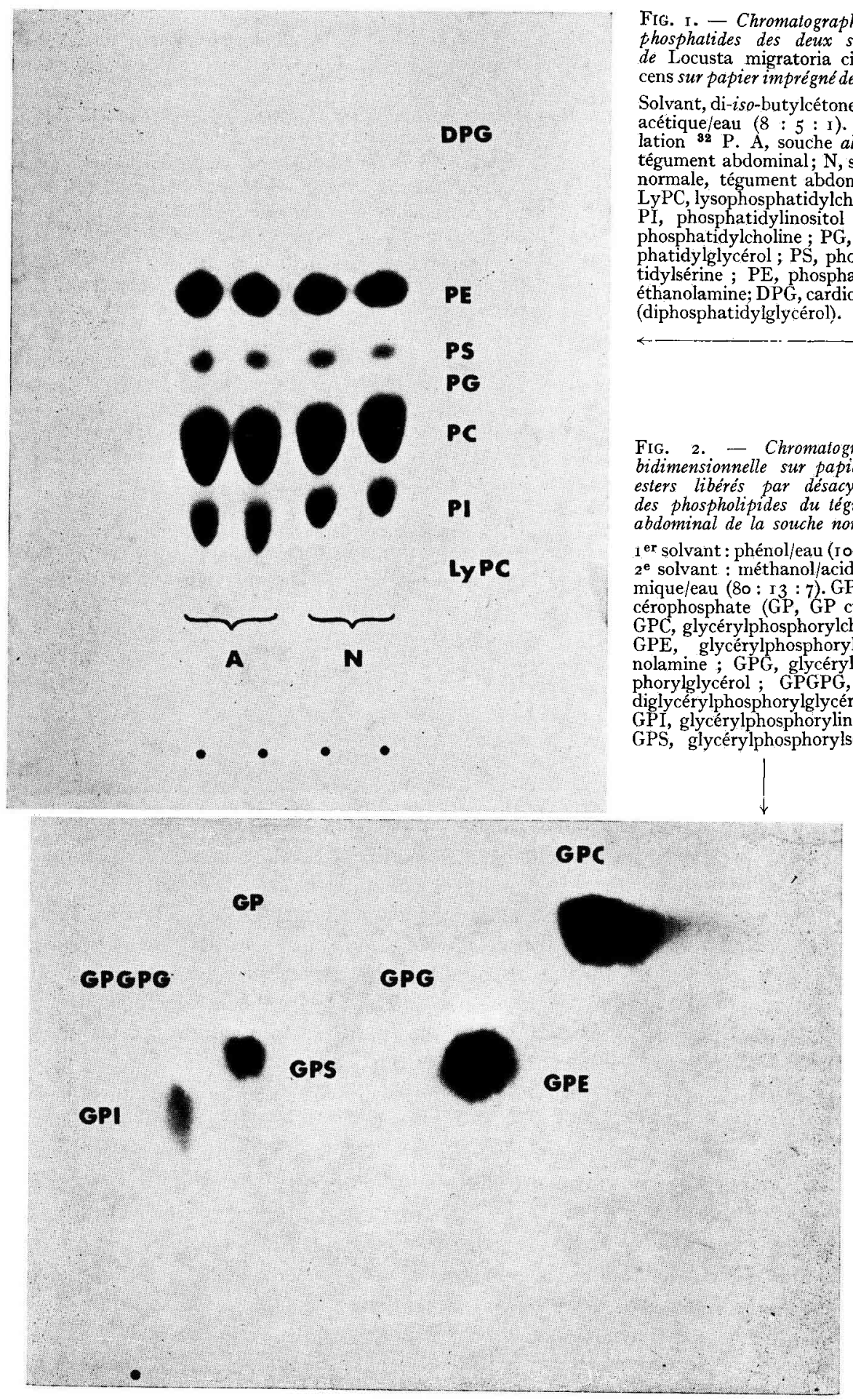




\section{TABLEAU I}

Phosphatiles du tégument abdominal des deux souches de Locusta migratoria cinerascens Abréviations identiques à celle de la figure 2

\begin{tabular}{|c|c|c|c|c|c|c|}
\hline \multirow{2}{*}{$\begin{array}{l}\text { Numéro } \\
\text { des taches }\end{array}$} & \multirow{2}{*}{$\begin{array}{l}\text { Coloration } \\
\text { Rhodamine } \\
\quad 6 G\end{array}$} & \multicolumn{2}{|c|}{ P. 100 de phosphose } & \multicolumn{2}{|c|}{ Produit de désacylation } & \multirow{2}{*}{$\begin{array}{l}\text { Identification } \\
\text { des phosphatides }\end{array}$} \\
\hline & & $\begin{array}{c}\text { Souche } \\
\text { normale }\end{array}$ & $\begin{array}{l}\text { Souche } \\
\text { albinos }\end{array}$ & $\begin{array}{c}\mathrm{Rf} \\
\text { phénol/eau }\end{array}$ & $\begin{array}{l}\text { identifié } \\
\text { comme }\end{array}$ & \\
\hline 1 & jaune & traces & traces & 0,89 & GPC & $\begin{array}{l}\text { Lysophosphatidyl- } \\
\text { choline. }\end{array}$ \\
\hline 2 & bleu & 14,1 & 14,6 & $\begin{array}{l}0,12 \\
0,26\end{array}$ & $\begin{array}{l}\text { GPI } \\
\alpha-G P\end{array}$ & Phosphatidvinositol. \\
\hline 3 & rose & 45,6 & 43,2 & $\begin{array}{l}0,89 \\
0,49\end{array}$ & $\begin{array}{l}\text { GPC } \\
\text { GP cycl. }\end{array}$ & $\begin{array}{l}\text { Phosphatidylcholine } \\
\text { (diester et acétal). }\end{array}$ \\
\hline 4 & bleu & traces & traces & 0,40 & GPG & Phosphatidylglycérol. \\
\hline 5 & bleu & 6,6 & 6,6 & 0,23 & GPS & $\begin{array}{l}\text { Phosphatidylsérine } \\
\text { (diester et acétal). }\end{array}$ \\
\hline 6 & lose & 23,9 & 25,5 & 0,59 & GPE & $\begin{array}{l}\text { Phosphatidyléthanol- } \\
\text { amine (diester et } \\
\text { acétal). }\end{array}$ \\
\hline 7 & bleu & 4,0 & 3,9 & $\begin{array}{l}0,15 \\
0,26\end{array}$ & $\begin{array}{l}\text { GPGPG } \\
\alpha-G P\end{array}$ & $\begin{array}{l}\text { Cardiolipide (diphos: } \\
\text { phatidylglycérol). }\end{array}$ \\
\hline
\end{tabular}

TABLEAU 2

Phosphatides de l'ail des deux souches de Locusta migratoria cinerascens

\begin{tabular}{|c|c|c|}
\hline \multirow{2}{*}{ Phosphatides } & \multicolumn{2}{|c|}{ P. 100 de phosphore } \\
\hline & Souche albinos & Souche normale \\
\hline Lysophosphatidylcholine ....... & traces & traces \\
\hline Phosphaiidylinositol ........... & 6,9 & 6,8 \\
\hline $\begin{array}{r}\text { Phosphatidylcholine (diester et } \\
\text { acétal) } \ldots \ldots \ldots \ldots \ldots \ldots \ldots\end{array}$ & 50,9 & 52,7 \\
\hline Phosphatidylglycérol $\ldots \ldots \ldots \ldots$. & traces & traces \\
\hline Phosphatidylsérine (diester et acétal) & 2,4 & 2,8 \\
\hline $\begin{array}{c}\text { Phosphatidyléthanolamine (diester } \\
\text { et acétal) } \ldots \ldots \ldots \ldots \ldots \ldots \ldots\end{array}$ & 33,4 & 33,4 \\
\hline $\begin{array}{l}\text { Cardiolipide } \text { (diphosphatidylgly- } \\
\text { cérol) } . . . \ldots \ldots \ldots \ldots \ldots \ldots \ldots \ldots\end{array}$ & 3,2 & 2,1 \\
\hline
\end{tabular}


effet de dilution intervient et que les dissemblances seraient plus flagrantes au niveau de chaque ultrastructure cellulaire à participation phospholipoprotéique. C'est ce que nous nous proposons d'élucider au cours de recherches ultérieures en isolant des fractions pures de granules pigmentaires.

\section{DISCUSSION}

Les différences discrètes entre souches normales et albinos de Locusta sont à rapprocher des constatations de FAST et BROWN ( 1962$)$ sur les lignées sauvages et lignées résistantes au DDT de Aedes aegypti et de BRIDGES, Crone et BEARD (I962) sur des lignées de $M$ usca domestica sensibles et résistantes à la dieldrine.

Les proportions des divers phospholipides relevées chez les Locusta adultes sont peu différentes de celles rapportées par ALIAIs et al. (I964) sur le même matériel en fin de développement embryonnaire, hormis en ce qui concerne le phosphatidylinositol, le cardiolipide et la lysophosphatidylcholine. Ceci s'accorde avec le fait que KinSELLA (Ig66) ne trouve pas de grandes différences entre l'embryon et 1'adulte de Periplaneta americana.

\section{TABLEAU 3}

Tableau comparatif de la composition de la fraction phospholipidique chez divers insectes, Les chiffres entre parenthèses correspondent aux renvois bibliographiques suivants : (I), FAST, I966 ;

(2), Bieber et $a l$., ig6r ; (3) Fast et Brown, ig6r ; (4) Crone et Bridges, Ig6r ; (5) Allen et Newburgh, I965; (6) Hashimoto et Mukai 1966 ; (7) Chojnacki et Korzybski 1962 ; (8) Tho-

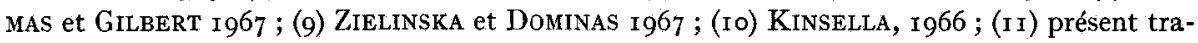
vail (*moyenne des résultats obtenus dans les 2 souches), (12) FAST, I967 ; (г3) Marinetri, ERBLAND et KOCHEN, I957.

\begin{tabular}{|c|c|c|c|c|c|c|c|}
\hline \multirow{2}{*}{ Espèces } & \multirow{2}{*}{$\begin{array}{l}\text { Lécithine } \\
\text { PC }\end{array}$} & \multicolumn{2}{|c|}{ Céphalines } & \multirow{2}{*}{ PI } & \multirow{2}{*}{ PG } & \multirow{2}{*}{ DPG } & \multirow{2}{*}{ LPC } \\
\hline & & $\mathrm{PE}$ & PS & & & & \\
\hline (2) Phormia regina ..... & 25 & 60 & 10 & 1 & 0 & & \\
\hline 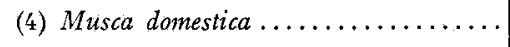 & 17,2 & 65,2 & 3,5 & 3,2 & 0 & 5,0 & \\
\hline (6) Ceroplastes pseudococciferus .......... & 25,3 & 55,6 & 3 & & & 2,1 & 0,6 \\
\hline (7) Arctia caja & 47 & 24 & 2 & 1,8 & 0 & traces & \\
\hline (8) Hyalophora cecropia & 43,1 & 27,3 & 3,9 & 2,0 & 0 & 9,1 & \\
\hline (10) Periplaneta americana & 60 & \multicolumn{2}{|c|}{31} & + & & \multicolumn{2}{|c|}{$3,1(+\mathrm{PI})$} \\
\hline \multirow{2}{*}{ (11) Locusta migratoria \{} & 44 & 24 & 7 & 15 & + & 3,8 & + \\
\hline & 50 & 33 & 2,5 & 7 & + & 2,6 & + \\
\hline $\begin{array}{l}\text { (13) Vertébré (cœur de porc) pour comparai- } \\
\text { son..................................... }\end{array}$ & 40,4 & 21,1 & 3,6 & & + & 11,4 & + \\
\hline
\end{tabular}




\section{TABLEAU 4}

Tableau comparatif de la valeur du rapport

phosphatidylcholine/phosphatidyléthanolamine (PC/PE) à travers les Ordres d'insecies étudiés Sources bibliographiques identiques à celles du tableau 3

\begin{tabular}{|c|c|c|c|c|c|}
\hline Ordre & Famille & Espèces & $\mathrm{PC}$ & $\mathrm{PE}$ & $\mathrm{PC} / \mathrm{PE}$ \\
\hline Diplìres & $\begin{array}{l}\text { Anthomyiidae } \\
\text { Calliphoridae } \\
\text { Chironomidae } \\
\text { Culicidae } \\
\text { Muscidae } \\
\text { Otalidae } \\
\text { Sarcophagidae } \\
\text { Tipulidae } \\
\text { Trypetidae } \\
\text { Cecidomyiidae }\end{array}$ & $\begin{array}{l}\text { (1) Hylemyia antiqua } \\
\text { (2) Phormia regina } \\
\text { (1) Chironomus sp. } \\
\text { (3) Aedes aegypti } \\
\text { (4) Musca domestica } \\
\text { (1) \} } \\
\text { (1) Euxesta notata } \\
\text { (5) Sarcophaga bullata } \\
\text { (1) Nephrotoma sodalis } \\
\text { (1) Strauzia longipsnnis } \\
\text { (1) Phytophaga rigidae } \\
\text { (1) Rhabdophaga swainei }\end{array}$ & $\begin{array}{l}17,4 \\
25,0 \\
26,1 \\
28,0 \\
17,2 \\
13,9 \\
17,5 \\
21,0 \\
26,0 \\
29,0 \\
37,5 \\
48,1\end{array}$ & $\begin{array}{l}52,9 \\
60,0 \\
46,5 \\
62,0 \\
65,2 \\
58,8 \\
53,4 \\
51,0 \\
46,5 \\
47,3 \\
38,6 \\
44,2\end{array}$ & $\begin{array}{l}0,34 \\
0,41 \\
0,56 \\
0,45 \\
0,26 \\
0,23 \\
0,33 \\
0,41 \\
0,56 \\
0,61 \\
1,02 \\
1,08\end{array}$ \\
\hline Homoptères & $\begin{array}{l}\text { Aphididae } \\
\text { Coccidae } \\
\text { Cercopidae }\end{array}$ & $\begin{array}{l}\text { (1) Anuraphis bakeri } \\
\text { (1) Prociphilus tesselatus } \\
\text { (1) Schizolachnus pi vi radiatae } \\
\text { (6) Ceroplastes pseudococciferus } \\
\text { (1) Aphrophora parallela }\end{array}$ & $\begin{array}{l}27,2 \\
29,7 \\
33,7 \\
25,3 \\
46,0\end{array}$ & $\begin{array}{l}40,8 \\
49,1 \\
54,3 \\
55,6 \\
28,7\end{array}$ & $\begin{array}{l}0,66 \\
0,60 \\
0,62 \\
0,46 \\
1,60\end{array}$ \\
\hline Coléoptères & $\begin{array}{l}\text { Chrysomelidae } \\
\text { Curculionidae } \\
\text { I'enebrionidae }\end{array}$ & $\begin{array}{l}\text { (1) Altica ambiens alni } \\
\text { (1) Chrysomela crotchi } \\
\text { (1) Pissodes strobi } \\
\text { (1) Tenebrio molitor }\end{array}$ & $\begin{array}{l}34,3 \\
39,8 \\
36,0 \\
39,8\end{array}$ & $\begin{array}{l}57,0 \\
38,2 \\
36,0 \\
41,7\end{array}$ & $\begin{array}{l}0,9 i \\
1,0 ; \\
1,00 \\
0,95\end{array}$ \\
\hline Lépidoplères & $\begin{array}{l}\text { Arctiidae } \\
\text { Geometridae } \\
\text { Notodontidae } \\
\text { Saturnidae } \\
\text { Tortıicidae }\end{array}$ & $\begin{array}{l}\text { (1) Arctia caja } \\
\text { () Hyphantria cunea } \\
\text { (1) Erannis tiliaria } \\
\text { (1) Paleacrita vernata } \\
\text { (1) Datana integerrima } \\
\text { (8) Hyalophora cecropia } \\
\text { (1) Archips cerasivoranus }\end{array}$ & $\begin{array}{l}47,0 \\
40,0 \\
45,4 \\
48,7 \\
37,6 \\
43,1 \\
45,8\end{array}$ & $\begin{array}{l}24,0 \\
31,8 \\
32,3 \\
25,8 \\
33,4 \\
27,3 \\
\varepsilon 9,5\end{array}$ & $\begin{array}{l}1,95 \\
1,26 \\
1,40 \\
1,89 \\
1,16 \\
1,58 \\
1,55\end{array}$ \\
\hline Hyménoptères & $\begin{array}{l}\text { Diprionidae } \\
\text { Tenthredinidae }\end{array}$ & $\begin{array}{l}\text { (1) Monoclemus iumiperinus } \\
\text { (') Neodiprion sertifer } \\
\text { (9) Acantholyda nemoralis } \\
\text { () Arge pecloralis } \\
\text { (1) Pikonema alaskensis }\end{array}$ & $\begin{array}{l}52,1 \\
49,2 \\
54,6 \\
41,6 \\
47,8\end{array}$ & $\begin{array}{l}28,2 \\
26,1 \\
37,4 \\
29,2 \\
30,3\end{array}$ & $\begin{array}{l}1,85 \\
1,88 \\
1,46 \\
1,43 \\
1,58\end{array}$ \\
\hline Dicloyplères & & (:c) Periplaneta americana & 60,0 & 31,0 & 1,93 \\
\hline Orthoptìres & $\begin{array}{l}\text { Acrididae } \\
\text { Gryllidae }\end{array}$ & $\begin{array}{l}\text { (11) Locusta migratoria* } \\
\text { (12) Gryllus bimaculatus }\end{array}$ & $\begin{array}{l}44,0 \\
54,2\end{array}$ & $\begin{array}{l}24,0 \\
35,4\end{array}$ & $\begin{array}{l}1,84 \\
1,53\end{array}$ \\
\hline Phasmoptères & & (1) Diapheromera femorata & 57,4 & 35,5 & 1,62 \\
\hline
\end{tabular}

Annales de Biologie animale. - 1969. 
Malgré les données bibliographiques fragmentaires sur ce point (tabl. 3), on peut dire que, par la composition de sa fraction phospholipidique, Locusta migratoria se distingue peu de la majorité des insectes (voir les revues de TrETZ, I96I ; FAST, I964 et I966; KINSELLA, I965) et des autres animaux.

La différence la plus notable concerne le pourcentage du phosphatidylinositol, très élevé chez Locusta, alors qu'il est généralement compris entre 2 et $3 \mathrm{p}$. Ioo chez la plupart des insectes (TIETZ, I965).

On peut, à la suite de FAST (rg66), classer les insectes étudiés à ce point de vue en considérant seulement le rapport caractéristique de la quantité de phosphatidylcholine à la quantité de phosphatidyléthanolamine (tab1. 4). On s'aperçoit alors que les Insectes se rangent en deux catégories bien tranchées :

$\mathrm{I}^{\mathrm{o}}$ ceux pour lesquels le rapport $\mathrm{PC} / \mathrm{PE}$ est très nettement inférieur à $\mathrm{I}, 00$; ce qui est le cas des Diptères (exceptée la famille des Cecidomyiidae) et des Homoptères connus (exceptée la famille des Cercopidae) ;

$2^{\circ}$ ceux pour lesquels ce rapport est soit très légèrement inférieur à $x, 00$ comme chez quelques Coléoptères, soit très nettement supérieur sans d'ailleurs atteindre 2,00 ; ce qui est le cas de tous les autres ordres d'Insectes et aussi des Vertébrés. Locusta se range dans cette dernière catégorie, comme les autres Orthoptéroïdes étudiés.

Les conséquences structurales et métaboliques de la valeur de ce rapport sont encore inconnues, mais il est logique de penser qu'elles sont importantes (FAST, I966).

Reçu pour publication en juillet 1968.

\title{
SUMMARY
}

\author{
GLYCEROPHOSPHATIDES IN " LOCUSTA AMIGRATORIA CINERASCENS " (FABR.) \\ AND ITS " ALBINO " MUTANT (ORTHOPTERA, ACRIDIDAE). \\ COMPARISON WITH OTHER INSECTS
}

The tegument and the eye of Locusta migratoria cinerascens FABR. were analyzed for phospho. lipids by ${ }^{32} \mathrm{P}$ sodium-orthophosphate labeling $(2,5 \mathrm{mCi}$ for 3 o animals).

Phospholipids were extracted in chloroform/methanol solvent; the non-lipid remainder was eliminated by FoLch's method (I957). The solution was chromatographed according to MARINETTI method (I957) in diisobutyl-cetone/acetic acid/water solvent, and spots were localized on chromatograms according to various classical techniques. Deacylations were performed for control according to BENSON's method. The glycerophosphatide rates were determined by radioactivity estimation of chromatogram spots. Autoradiochromatographies were performed (fig. I and 2).

There were no significant differences between the two strains. The average phospholipid distribution was as follows: (tables $\mathrm{I}$ and $\mathbf{2}$ ).

- 44 per cent phosphatidycholine (v. $5^{\mathrm{I}}$ per cent in the eye).

- 24 per cent phosphatidyl ethanolamine (v. 33 per cent in the eye).

- I4 per cent phosphatidylinositol ( $v .7$ per cent in the eye).

- 6,5 per cent phosphatidylserine (v, 2.5 per cent in the eye).

- Slight traces of phosphatidylglycerol and lyso-phosphatidylcholine were detected.

Plasmalogens were not substracted from nitrogenous phospholipids. The rate of phosphatidylinositol is higher in Locusia than in other studied insects (table 3).

The phosphatidylcholine/phosphatidylethanolamine ratio was 1.84 , in Locusta, which is in accordance with the majority of insects (with the exception of the Diptera and Homoptera) for which the ration ranges between $I, 0$ and 2.0 (table 4 ). 


\section{RÉFÉRENCESS BIBLIOGRAPHIQUES}

ALBRECHT G., Untersuchungen über die chemische Zusammensetzung einiger Insektenfette. Z. vergleich. Physiol., 44, 487-508.

alzais J.-P., Bergerard J., Étienne J., Polonovski J., i 964 . Nature et évolution des lipides au cours de l'embryogenèse de Locusta migratoria migratorioides L. J. Ins. Physiol., 10, 753-772.

Allen R. R., Newburch R. W., 1965. Phospholipid composition of fat bodies of Sarcophaga bullata. J. Ins. Physiol., 11, 1601-1603.

Benson A. A., Maruo B., I958. Plant phospholipids. I. Identification of the phosphatidylglycerols. Biochim. Biophys. Acta., 2\%, I89-195.

Breber L. L., Hodgson E., Cheldelin V. H., Brookes V. J., Newburgh R. W., I96i. Phospholipid patterns in the blowfly Phormia regina (MEIGEN). J. Biol. Chem., 236, 2590-2595.

Bouthrer A., 1966. Modifications des pigments (ommochromes et ptérines) en relation avec la mutation albinos chez Locusta migratoria cinerascens FABR. (Orthoptères, Acrididae). C. R. Acad. Sci., 262 D, 1480I 483 .

Bridges R. G., Crone H.D, Beard J.R., I 662 . A study of the phospholipids of dieldrin-resistant and susceptible houseflies, with particular reference to those of the thoracic ganglion. in Radioisotopes and radiation in entomology. International atomic agency. Vienna. $\mathrm{I}_{45} \mathrm{-1} 53$.

ChojNAcki T., KoRzYBsKi T., I962. Biosynthesis of phospholipids in insects. III. The incorporation of 32P-orthophosphate into phospholipids of Arctia caia moths. Acta biochim. Polon., 9, 95-1 10.

Crone H. D., Bridges R. G., I963. The phospholipids of the housefly, Musca domestica. Biochem. J., 89, I I-2I.

Dallner G., Siekevitz P., Palade G. E., I 966 . Biogenesis of endoplasmic reticulum membranes. I. Structural and chemical differenciation of developing rat hepatocyte. J. Cell Biol., 30, 73-96.

Douce R., I964. Identification et dosage de quelques glycérophosphatides dans des souches normales et tumorales de scorsonère cultivées in vitro. C. R. Acad., Sci., 259, 3066-3069.

FAST P. G., I964. Insect lipids : a review. Mem. entomol. Soc. Canad. $\mathrm{n}^{\circ}$ 37, I I p.

FAST P. G., 1966. A comparative study of the phospholipids and fatty acids of some insects. Lipids, 1, 200-215.

Fast P. G., I 967. An analysis of the lipids of Gryllus bimaculatus (DE GEER). (Insecta. Orthoptera). Canad. J. Biochem., 45, 503-506.

FAST P. G., Brown A. W. A., 1962. Lipids of DDT-resistant and susceptible larvae of Aedes aegypti. Ann. entomol. Soc. Amer., 55, 663-672.

Forch J., LeEs M., StOANe Stanley G. H., t957. A simple method for the isolation and purification of total lipids from animal tissues. J. Biol. Chem., 228, 497-509.

Fuge H., 1966. Uber die Bildung der Ommochromgrana von Drosophila melanogaster. Naturwissensch. 53, г 36.

Hashimoto A., Mukai K., ig66. Étude des lipides des Coccidae. IV. Les phospholipides de Ceroplastes pseudococciferus (GREEN), en japonais, résumé angl. J. Agric. chem. Soc. Jap., 40, 330-340.

Kinsella J. E., I g66. Phospholipid patterns of Periplaneta americana during embryogenesis. Comp. Biochem. Physiol., 17, 635-640.

MarinetTI G. V., ERbLAND J., Kochen J., 1957. Quantitative chromatography of phosphatides. Fed. Proc., 16, 837-844.

Osman M. F. H., Schmidt G. H., I 96I. Analyse der Körperfette von imaginalen Wanderheuschrecken der Art Locusta migratoria migratorioides L. (Orth.). Biochem. Z., 334, 441-450.

Shoup J. R., I 966 . The development of pigment granules in the eyes of wild type and mutant Drosophila melanogaster. J. Cell Biol., 29, 223-249.

K. K., GiLBert L. I., 1967. Phospholipid synthesis during flight muscle development in the American silkmoth Hyalophora cecropia. Comp. Biochem. Physiol., 21, 279-29o.

TIETZ A., 1965. Metabolic pathways in the Insect fat body. in Handbook of Physiology (American Physiological Society). Chap. 7 (Adipose Tissue) 45-54.

VERDIER M., 1965. De la mutation albinos de Locusta migratoria. I. Origine et description morphologique. Bull. Soc. zool. Fr., 80, 493-501.

ZIELINSKa Z. M., Dominas A., 1967. The origin of phospholipid ethanolamine and choline in a sawfly. Acantholyda nemoralis. J. Ins. Physiol., 13, 1769-1 779 . 